\title{
Rapamycin, anti-aging, and avoiding the fate of Tithonus
}

\author{
Arlan Richardson \\ Barshop Institute for Longevity and Aging Studies, University of Texas Health Science Center at San Antonio, \\ and Geriatric, Research, Education and Clinical Center, South Texas Veterans Health Care System, San Antonio, Texas, USA.
}

\begin{abstract}
The discovery that rapamycin increased the lifespan of mice was recognized by Science as one of the top 10 scientific breakthroughs of 2009. In addition to increasing lifespan, Neff and colleagues show that while rapamycin improves several functions/pathologies that change with age, it has little effect on the majority of the physiological and structural parameters they evaluated. What do these data tell us about the ability of rapamycin to delay aging and improve quality of life, i.e., prevent the fate of Tithonus?
\end{abstract}

\section{The Tithonus effect}

The consequences of longevity without health and vigor are dramatically portrayed in the Greek myth about Tithonus, a prince of Troy. After the goddess Eos kidnapped him, she asked Zeus to make Tithonus immortal. Unfortunately, Eos forgot to ask for eternal youth.

But when loathsome old age pressed full upon [Tithonus], and be could not move nor lift his limbs, this seemed to [Eos] in her heart the best counsel: she laid him in a room.... There he babbles endlessly, and no more has strength at all ....

-Homeric Hymn to Aphrodite V, translated by Hugh G. Evelyn-White

In 2009, Harrison et al. (1) reported that rapamycin (also known as sirolimus) increased both mean and maximum lifespan of mice, demonstrating that all competing causes of mortality (i.e., age-related diseases) are delayed and suggesting that rapamycin slows aging. Because rapamycin is approved by the FDA for transplant and cancer patients, this discovery has enormous translational potential; however, before taking a potential anti-aging therapy to humans, it is necessary to demonstrate that the agent does not produce a "Tithonus phenotype," where the increase in lifespan is accompanied by more disability and disease and a greater loss of physiological functions, i.e., a reduced quality of life.

Conflict of interest: Arlan Richardson holds stock in Rapamycin Holdings Inc.

Citation for this article: J Clin Invest. 2013; 123(8):3204-3206. doi:10.1172/JCI70800.

\section{A comprehensive assessment of aging in mice}

In this issue of the JCI, Neff et al. (2) describe the results from their large-scale study of the effect of rapamycin on more than 150 aging phenotypes across 25 different tissues in male C57BL/ 6 mice. They identified several aging phenotypes that were improved by rapamycin, in particular behavior/cognition, several immune parameters, and a number of pathological lesions. The effect of rapamycin on cognition is particularly important in an aging context because this physiological domain is critical for the quality of life in humans. Neff et al. (2) showed that learning and memory were improved. Halloran et al. (3) and Majumder et al. (4) also found that rapamycin improved the performance of old mice. Furthermore, rapamycin was previously observed to restore memory in transgenic mouse models of Alzheimer's disease (5-7). Thus, the current data indicate that rapamycin has a robust effect on cognitive performance in mice.

Neff et al. (2) found that the vast majority of parameters they measured were not significantly altered by rapamycin, including vision, hearing, and cardiac and skeletal muscle function, all of which are important to quality of life. Rapamycin has been found to improve cardiac function in old mice (P. Rabinovitch, personal communication) and in Lmna ${ }^{-1-}$ mice (8). While Neff et al. (2) saw no effect of rapamycin on muscle function using measurements of grip strength and rotarod performance (a measure of muscle function as well as balance and motor coordination), Zhang et al. (9) found that rotarod performance and gait improved significantly in old male and female mice given rapamycin. Rapamy- cin also improved rotarod performance in mouse models of muscular dystrophy (8) and Huntington disease (10). Wilkinson et al. (11) observed that rapamycin reduced the stiffening and loss of elasticity in tendons in old mice. Taken together, the conflicting data from previous studies suggests that it is too early to conclude that rapamycin has no effect on all cardiac and muscle functions that decline with age.

The discrepancies in the functional data could arise from several factors. First, many of the functional assays show a great deal of laboratory-to-laboratory variation in the equipment and protocols used. Second, as Neff et al. (2) note, differences could also arise from differences in mouse strains. Third, it is becoming increasingly apparent that rapamycin shows differences in how it affects various parameters in male and female mice (9). Fourth, the effect of rapamycin on a function could be influenced by the length of treatment, the age at which rapamycin treatment was initiated, and the delivery method.

The increase in the severity and number of diseases is a hallmark of aging; therefore, an important criterion for evaluating whether a manipulation increases lifespan by delaying aging is the appearance and severity of pathological lesions and disease. Dietary restriction, the most well-studied anti-aging manipulation, has been shown to delay and/or reduce most age-related pathologies in rodents (12). Neff et al. (2) conducted a comprehensive histopathological analysis of old mice and found that precancerous lesions were significantly reduced by rapamycin, while other age-related pathological lesions (including cataracts, which are often used as a measure of aging) were not significantly altered. Wilkinson et al. (11) reported that rapamycin reduced a number of histopathology endpoints in the heart, liver, adrenal glands, and endometrium in old mice; however, cataracts were increased. Of particular interest is the effect of rapamycin on end-of-life pathology, including cause of death. In two separate 
studies using genetically heterogenous mice (UM-HET3), rapamycin did not appear to change the cause of death $(1,13)$. Zhang et al. (9) also observed no difference in most end-of-life pathology or cause of death in male and female C57BL/6 mice, except for a reduced number of neoplastic lesions and adenoma in female mice fed rapamycin. Thus, the current data show that increased longevity is associated with little change in end-of-life pathology. Because cancer is the primary cause of death in UM-HET3 and C57BL/6 mice, Harrison et al. (1) and Neff et al. (2) have pointed out that the increased longevity could arise largely from reduced incidence of cancer. Recent studies with mice genetically engineered to develop specific types of cancer support this possibility, showing that rapamycin (or rapalogs) not only reduces the progression of a wide variety of tumors, it also increases the lifespan of mice (14-17).

The study by Neff et al. (2) is a tour de force; no one has previously conducted such a comprehensive analysis on the effect of a potential anti-aging manipulation on the functional status of an animal. Because the majority of age-sensitive parameters were not significantly improved by rapamycin, and because many of the parameters that were enhanced/improved by rapamycin were also altered in young mice, Neff et al. conclude that rapamycin has "limited effects on mammalian aging itself" (2), raising the concern of whether rapamycin extends healthspan as well as lifespan. Their study underscores the dilemma that investigators face when trying to determine whether a manipulation alters aging. Must all processes that change with age be reversed/improved by rapamycin? If so, would these changes have to be specific to older animals? Even dietary restriction, which is universally understood to delay aging, does not alter all age-sensitive functions and pathologies, many of which are altered in young animals (18). If not all processes have to be altered by rapamycin, what percent of age-related changes have to be enhanced or improved for rapamycin to be considered an anti-aging agent? Are some functions (memory and cardiac/muscle function) more important in improving/ maintaining quality of life than others?

\section{Rapamycin and the Tithonus phenotype}

I do not believe that the current data from Neff et al. (2) and others described above allow us to answer with any certainty the question of whether rapamycin increases longevity by delaying aging. The current data do allow us to answer an equally important question: Do mice fed rapamycin exhibit a Tithonus phenotype? In other words, does rapamycin extend lifespan in the absence of improved quality of life? First, there is no general increase in the pathology observed at death $(1,9$, 13); in fact, some pathologies appear to be reduced, i.e., the mice live longer without any increase in disease/pathology. Second, several important physiological functions are enhanced by rapamycin; however, it is unclear whether this is due to a delay in aging or an induction of function. On the other hand, there is no evidence that old mice fed rapamycin exhibit functional losses that could negatively impact quality of life. Third, several diseases in addition to cancer are delayed by rapamycin (or rapalogs): atherosclerotic plaque formation is reduced in mouse models mimicking human atherosclerosis (19); memory is improved and amyloid plaque deposition reduced in transgenic models of Alzheimer's disease (5-7); lifespan is increased and cardiac and muscle function improved in a mouse model of Hutchinson-Gilfords progeria syndrome (8); and muscle function is improved and huntingtin fragments are reduced in a transgenic mouse model of Huntington disease (10). Therefore, rapamycin appears to have a broad effect on preventing/delaying a large number of age-related diseases that are important in human health.

The current data demonstrate conclusively that mice fed rapamycin live three to four months longer (the equivalent of about a decade in human years) and exhibit the same health status/quality of life at time of death as mice that do not receive rapamycin. In fact, mice receiving rapamycin may show improved function in some physiological parameters as well as reduced incidence/severity of some age-related diseases; they do not exhibit the Tithonus phenotype. Neff et al. (2) observed two toxicities with rapamycin: nephrotoxicity and testicular degeneration. Wilkinson et al. (11) also observed testicular degeneration in mice fed rapamycin. However, neither Miller et al. (13) nor Zhang et al. (9) observed any evidence of increased renal pathology at end of life in either male or female mice fed rapamycin. Therefore, the only consistent toxicity observed in mice chronically fed rapamycin is testicular degeneration.
The toxicity/side effects of rapamycin in humans are best shown from clinical trials with cancer patients in which rapalogs are used as a monotherapy. Mammalian target of rapamycin (mTOR) inhibitors have specific and relatively consistent toxicities (e.g., hyperlipidemia, hyperglycemia, stomatitis, rash, and myelosuppression), which are rarely life threatening or serious $(20,21)$. The rare occurrence of interstitial pneumonitis is a potentially serious toxicity that can be resolved by discontinuing treatment. When used as a monotherapy, rapamycin and rapalogs do not appear to be immunosuppressive in humans (20) or mice (22). Based on their experience using rapalogs to treat cancer, Mita and Mita (23) conclude that patients treated with rapalogs do "remarkably well for prolonged time with almost no change in the quality of life." Because the toxicity profile of rapamycin is well established, the use of rapamycin or rapalogs is relatively safe and compatible with prolonged use in patients at risk for specific diseases. For example, clinical trials are currently being conducted to test the efficacy of rapalogs in preventing the reoccurrence of cancer in renal and breast cancer patients. In sum, I believe that the importance of the study by Neff et al. (2) is that the data support the feasibility of clinical trials to study the efficacy of rapamycin in treating diseases of the elderly, especially those that are debilitating and for which no current treatment is known, such as Alzheimer's disease and other neurodegenerative diseases.

\section{Acknowledgments}

The author acknowledges the assistance of Corinne Price in writing this commentary and NIH RC2 Grand Opportunity grant (RC2 AG036613) for support of his research on rapamycin.

Address correspondence to: Arlan Richardson, Barshop Institute, University of Texas Health Science Center at San Antonio, 7703 Floyd Curl Drive, San Antonio, Texas 78229-3900, USA. Phone: 210.562.6140; Fax: 210.562.6150; E-mail: richardsona@ uthscsa.edu.

1. Harrison DE, et al. Rapamycin fed late in life extends lifespan in genetically heterogeneous mice. Nature. 2009;460(7253):392-395.

2. Neff F, et al. Rapamycin extends murine lifespan but has limited effects on aging. J Clin Invest. 2013;123(8):3272-3291.

3. Halloran J, et al. Chronic inhibition of mTOR by rapamycin modulates cognitive and non-cognitive components of behavior throughout lifespan in mice. Neuroscience. 2012;223:102-113. 
4. Majumder S, et al. Life-long rapamycin administration ameliorates age-dependent cognitive deficits by reducing IL-1 $\beta$ and NMDA signaling. Aging Cell. 2012;11(2):326-335.

5. Caccamo A, Majumder S, Richardson A, Strong $\mathrm{R}$, Oddo S. Molecular interplay between $\mathrm{mTOR}$, amyloid-beta, and tau: Effects on cognitive impairments. J Biol Chem. 2010;285(17):13107-13120

6. Spilman $S$, et al. Inhibition of mTOR by rapamycin abolishes cognitive deficits and reduces amyloid- $\beta$ levels in a mouse model of Alzheimer's disease. PLoS One. 2010;5(4):e9979.

7. Majumder S, Richardson A, Strong R, Oddo S. Inducing autophagy by rapamycin before but not after plaques and tangles formation ameliorates cognitive deficits in a mouse model of Alzheimer's disease. PLoS One. 2011;6(9):e25416.

8. Ramos FJ, et al. Rapamycin reverses elevated mTORC1 signaling in lamin A/C-deficient mice, rescues cardiac and skeletal muscle function, and extends survival. Sci Transl Med. 2012;4(144):144ra103.

9. Zhang Y, et al. Rapamycin extends life and health in C57BL/ 6 mice [published online ahead of print May 16, 2013]. J Gerontol A Biol Sci Med Sci. doi:10.1093/gerona/glt056.

10. Ravikumar B, et al. Inhibition of mTOR induces autophagy and reduces toxicity of polyglutamine expansions in fly and mouse models of Huntington disease. Nat Genet. 2004;36(6):585-595.

11. Wilkinson JE, et al. Rapamycin slows aging in mice. Aging Cell. 2012;11(4):675-682.

12. Ikeno Y, Lew CM, Cortez LA, Webb CR, Lee S, Hubbard GB. Do long-lived mutant and calorierestricted mice share common anti-aging mechanisms? - a pathological point of view. Age (Dordr). 2006;28(2):163-171.

13. Miller RA, et al. Rapamycin, but not resveratrol or simvastatin, extends lifespan of genetically heterogeneous mice. J Gerontol A Biol Sci Med Sci. 2011;66(2):191-20.

14. Fujishita T, Aoki K, Lane HA, Aoki M, Taketo MM. Inhibition of the mTORC1 pathway suppresses intestinal polyp formation and reduces mortality in ApcDelta716 mice. Proc Natl Acad Sci U S A. 2008;105(36):13544-13549.

15. Comas M, et al. New nanoformulation of rapamycin Rapatar extends lifespan in homozygous $p 53^{--}$ mice by delaying carcinogenesis. Aging (Albany NY). 2012;4(10):715-722.

16. Anisimov VN, et al. Rapamycin extends maximal lifespan in cancer-prone mice. Am J Pathol. 2010;175(5):2092-2097.
17. Livi CB, et al. Rapamycin extends life span of $\mathrm{Rb} 1^{+/}$ mice by inhibiting neuroendocrine tumors. Aging (Albany NY). 2013;5(2):100-110.

18. Richardson A, McCarter R. Mechanism of food restriction: change of rate or change of set point? In: Ingram DK, Baker GT, Shock NW, eds. The Potential for Nutritional Modulation of Aging Processes. Trumbull, Connecticut, USA: Food and Nutrition Press, Inc.; 1991:177-192.

19. Mueller MA, Beutner F, Teupser D, Ceglarek U, Thiery J. Prevention of atherosclerosis by the mTOR inhibitor everolimus in $\mathrm{LDLR}^{-/-}$mice despite severe hypercholesterolemia. Atherosclerosis. 2008;198(1):39-48.

20. Cohen EEW. mTOR: The mammalian target of replication. J Clin Oncol. 2008;26(3):348-349.

21. Scott A, Karnad S, Karnad A, Brenner AJ. Common toxicities of mammalian target of rapamycin inhibitors. Target Oncol. 2011;6(2):125-129.

22. Hinojosa CA, et al. Enteric-delivered rapamycin enhances resistance of aged mice to pneumococcal pneumonia through reduced cellular senescence. Exp Gerontol. 2012;47(12):958-965.

23. Mita M, Mita A. Are we ready to move away from nature? The rapamycin story. Target Oncol. 2011;6(2):63-64.

\title{
The future of molecular chaperones and beyond
}

\author{
Rona G. Giffard, ${ }^{1}$ Alberto J.L. Macario, ${ }^{2,3}$ and Everly Conway de Macario²
}

\begin{abstract}
1Department of Anesthesia, Stanford University School of Medicine, Stanford, California, USA. 2Department of Microbiology and Immunology, School of Medicine, University of Maryland at Baltimore, and IMET, Columbus Center, Baltimore, Maryland, USA.
\end{abstract} ${ }^{3}$ Istituto EuroMediterraneo di Scienza e Tecnologia (IEMEST), Palermo, Italy.

\begin{abstract}
Protection of hair cells by HSP70 released by supporting cells is reported by May et al. in this issue of the JCI. Their findings suggest a new way to reduce ototoxicity from therapeutic medications and raise larger questions about the role and integration of heat shock proteins in non-cell-autonomous responses to stress. Increasing evidence suggests an important role for extracellular heat shock proteins in both the nervous system and the immune system. The work also suggests that defective chaperones could cause ear disease and supports the potential use of chaperone therapeutics.
\end{abstract}

\section{Heat shock proteins}

Heat shock proteins (HSPs) are the product of heat shock genes, initially defined as genes induced by a short, sudden temperature elevation above normal; however, many HSP genes are induced by stressors other than heat shock, and some are constitutively expressed essential proteins. Initially, HSP70 was recognized as an intracellular chaperone with a central role in protein folding of nascent and denatured proteins (1-4), and extracellular HSP70 was thought to be the result of cell death. A body of work has now demonstrated that HSP70 is released from a wide

Conflict of interest: The authors have declared that no conflict of interest exists.

Citation for this article: J Clin Invest. 2013; 123(8):3206-3208. doi:10.1172/JCI70799. variety of cells via a noncanonical secretion pathway $(5,6)$, as the sequence is devoid of a consensus secretory signal, and inhibitors of the common secretory pathway fail to block secretion. HSP70 is thought to share secretion pathways used by other leaderless proteins that are nevertheless secreted, such as IL- $1 \beta$, but this remains an area of active research.

HSP70 is a highly evolutionarily conserved molecule, from bacteria to people. In humans, it has expanded from the single DNAK protein found in bacteria to a family of at least 17 members in humans (7). Further, some of the genes likely produce more than one mRNA and protein variant. Because it is such an ancient protein and because it has always been involved with stress sensing and stress resistance, it is not surprising that it also participates with two organ systems that have evolved to sense environmental challenges and respond to them, the nervous system and the immune system. Indeed, it is especially in these two systems that HSP70 roles beyond the classical intracellular locations have begun to be revealed.

HSP70 has been shown to have many paracrine effects, especially in the setting of injury and repair. Extracellular HSP70, both free and endosome/vesicle-associated, provides information about stress and cell injury. It acts as an alarmin or damageassociated molecular pattern; activates signaling cascades, including the cytokineinducing MyD88/IRAK/NF-кB pathway (8); regulates signaling pathways, including FOXO and the MAPK p38 (9); and acts on numerous cell types, including immune cells, epithelial cells, hepatocytes, and neurons. For example, HSP70 plays a central role in the regulation of inflammation and regeneration following muscle injury (9).

The article by May et al. reports that HSP70 release from supporting cells is necessary and sufficient to protect mechanosensory hair cell neurons from the toxic effects of aminoglycoside antibiotics (10). This is of broad importance, as there are 